\title{
Analyses of the Adsorption Structures of Friction Modifiers by Means of Quantitative Structure-Property Relationship Method and Sum Frequency Generation Spectroscopy
}

\author{
Hiroaki Koshima $^{1)^{*}}$, Hideki Kamano ${ }^{2)}$, Yoshio Hisaeda ${ }^{3)}$, Huijin Liu ${ }^{4)}$ and Shen $\mathrm{Ye}^{4)}$ \\ ${ }^{1)}$ Advanced Technology Research Laboratories, Idemitsu Kosan Co. Ltd. \\ 1280 Kamiizumi, Sodegaura, Chiba 299-0293, Japan \\ ${ }^{2)}$ Lubricants Research Laboratory, Idemitsu Kosan Co. Ltd. \\ 24-4 Anesakikaigan, Ichihara, Chiba 299-0107, Japan \\ ${ }^{3)}$ Department of Chemistry and Biochemistry, Graduate School of Engineering, Kyushu University \\ 744 Motooka, Nishi-ku, Fukuoka 819-0395, Japan \\ ${ }^{4)}$ Catalysis Research Center, Hokkaido University \\ North 21, West 10, Kita-ku, Sapporo, Hokkaido 001-0021, Japan \\ *Corresponding author: hiroaki.koshima@si.idemitsu.co.jp
}

( Manuscript received 1 February 2010; accepted 3 August 2010; published 15 September 2010 )

\begin{abstract}
Blending an optimum amount of friction modifiers into lubricant is one of the important measures to reduce fuel consumption induced by the frictional loss for automobiles. However, the agents containing metal or phosphorus compounds can cause catalyst poisoning and clogging in the filters of the exhaust gas refining system. Thus, development of metal-free and phosphorus-free agents with a long-term stability under real working condition is highly desirable. In the present work, the friction coefficients on the metal surface in lubricants containing different friction modifiers were investigated by the reciprocating friction test in detail. A statistical method based on the quantitative structure-property relationship (QSPR) analysis has been employed to correlate the chemical nature of additives with their effect on the friction reduction behaviors. An empirical equation relating the molecular structure and its friction reduction efficiency has been proposed as a standard for a good friction modifier. Furthermore, a surface-sensitive vibration spectroscopy, sum frequency generation (SFG) spectroscopy, has been used to analyze the molecular structures of the lubricants adsorbed on the metal surface. The SFG observation suggested that a certain relationship between the adsorption structure and friction reduction effect of these agents.
\end{abstract}

Keywords: fuel consumption, friction, surface, QSPR, SFG

\section{Introduction}

In recent years, environmental problems such as the global warming and the ozone depletion have drawn wide attentions. In particular, substantial research is being focused on reducing the emission of carbon dioxide, which is considered to significantly affect the global warming. Thus, there is an increased requirement to improve fuel consumption in the automotive industry in which lubricants play an important role. Blending an optimum amount of friction modifiers into lubricant is one of the general measures to reduce fuel consumption induced by the friction loss. It is known that the friction coefficient on the metal surface can be reduced by adding some molybdenum compounds such as molybdenum dithiocarbamate (MoDTC) and molybdenum dithio- phosphate (MoDTP) ${ }^{1-4)}$. However, these additives only show good friction-reducing effect in a fresh state and deteriorate quickly with time. In addition, the friction reduction performance cannot be sustained in the presence of soot ${ }^{5,6}$. These chemicals containing with metal or phosphorus compounds can also cause clogging of exhaust gas refining system as well as catalyst poisoning. Thus, development of metal-free and phosphorus-free agents with a long-term stability under real working conditions is strongly desirable.

In order to overcome these technical problems mentioned above, the friction reduction characteristics for a number of model amphiphilic molecules including 
aliphatic alcohols, aliphatic amines, fatty acids and oxygen- and nitrogen-based heterocyclic compounds, have been evaluated on the metal surface in the present work. Lundgren et al. investigated the friction forces between layers of the unsaturated fatty acids (stearic, oleic, linoleic and linolenic acid) with a surface forces apparatus. They found that the friction coefficient increased with increasing unsaturation ${ }^{7,8)}$. These results demonstrate that the friction features significantly depend on the nature of hydrophilic and/or lipophilic groups in these additives.

The quantitative structure-property relationship (QSPR) analysis has been tentatively employed to elucidate the relationships between the structures and the properties of these lubricant molecules ${ }^{9)}$. As reported previously, the QSPR method enabled the development of traction fluid for continuously variable transmission (CVT) vehicles ${ }^{10,11)}$. This method has also been applied to support the development of other additives for engine oil $^{12-15)}$. Based on the analysis, we are able to get an empirical equation correlating the molecular structure of these additives with their friction reduction features on the metal surface. The factor analysis of the QSPR method has been employed to determine the friction mechanism of friction modifiers on the metal surface. The present analysis revealed that better additive molecules always orientate their hydrophobic alkyl chain away from the metal surface. A suitable chemical structure for friction modifiers on the metal surface has been proposed based on the information.

On the other hand, since the structure of a molecule adsorbed on the solid surface is not necessarily identical with its structure in bulk phase, it is important to know the adsorption structures of these lubricant molecules on the metal surface. However, such structural information is still not available especially under the ambient conditions where the standard electron diffraction techniques developed in ultrahigh vacuum (UHV) environment do not work. In this regard, the vibrational spectroscopy can provide abundant information for the molecular structures. Unfortunately, the traditional vibrational spectroscopy techniques, such as infrared (IR) spectroscopy and Raman scattering, are not intrinsically surface-specific, and usually not sensitive enough to study monolayers. For example, the convention IR measurement is difficult to distinguish the molecular structure on the surface of a thin film since the film bulk can also give strong IR absorption ${ }^{16-18)}$.

By the way, sum frequency generation (SFG) takes advantage of a second-order non-linear optical effect and allows a selective vibrational spectroscopic observation on the surface or interface. This method has been employed to study the adsorption of many organic molecules on the solid surfaces ${ }^{17-24)}$. Duffy et al. investigated the adsorption of potassium oleate and sodium octanoate, two typical additives into lubricants, at the iron-water interface by SFG spectroscopy. They found that potassium oleate was adsorbed at the iron surface as an ordered bilayer while sodium octanoate was adsorbed as a completely disordered film. Based on these results, they proposed that the ordered adlayer of oleate is more effective at lubricating than that of octanoate $^{25)}$.

In the present study, to understand the lubrication mechanism of the friction modifiers, SFG is also employed to analyze the adsorption behavior of various additive molecules on the metal surface. The SFG results suggested that a certain relationship between the adsorbed structure of the additive molecules on the metal surface and their frication reduction performance evaluated by the friction coefficient measurements and the QSPR analysis.

\section{Experimental}

As shown in Figure 1, the friction features of lubricants on a polished iron plate (SUJ-2) has been carefully evaluated using a reciprocating friction tester TE77 (Phoenix Tribology Ltd.).

A number of additives, most of them are amphiphilic (Figure 2), were tested as additive into lubricant oil. The sample was prepared by 1.0 mass $\%$ in poly-alpha-olefin (PAO) with a viscosity of $17.3 \mathrm{~mm}^{2} / \mathrm{s}\left(40{ }^{\circ} \mathrm{C}\right)$ which mainly contains decene trimer and tetramer. In addition, each chemical was refined through Silicagel column, but oleyl group includes isomers of about 20 area \% by ELS Detector of HPLC.

After a 5-min running-in, the friction coefficients $(\mu)$ for each lubricant sample were measured at $100{ }^{\circ} \mathrm{C}$ under a load of $50 \mathrm{~N}$. Motion rate was gradually increased from $2.5 \mathrm{~Hz}$ to $5.0 \mathrm{~Hz}$. The other experimental conditions are given in Table 1.

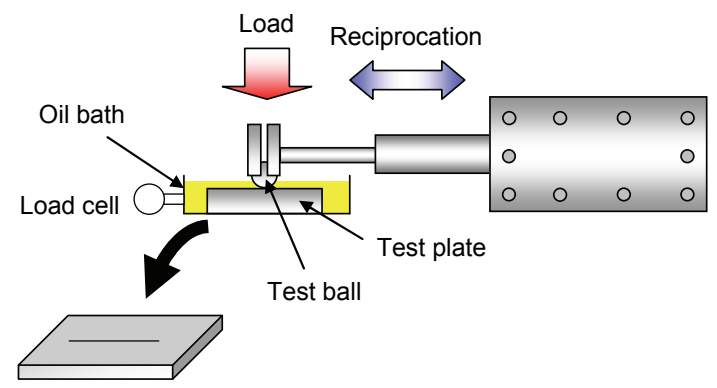

Figure 1 Schematic diagram of reciprocating friction tester

Table 1 Reciprocating friction test conditions

\begin{tabular}{lll}
\hline Test piece & Test plate & SUJ-2 \\
& Test ball & SUJ-2 $(\varphi 10 \mathrm{~mm})$ \\
\hline Test condition & Oil temperature & $100{ }^{\circ} \mathrm{C}$ \\
& Amplitude & $15 \mathrm{~mm}$ \\
& Load & $50 \mathrm{~N}$ \\
& Frequency & $2.5,3.0,3.5$, \\
& & $4.0,4.5,5.0 \mathrm{~Hz}$ \\
\hline
\end{tabular}



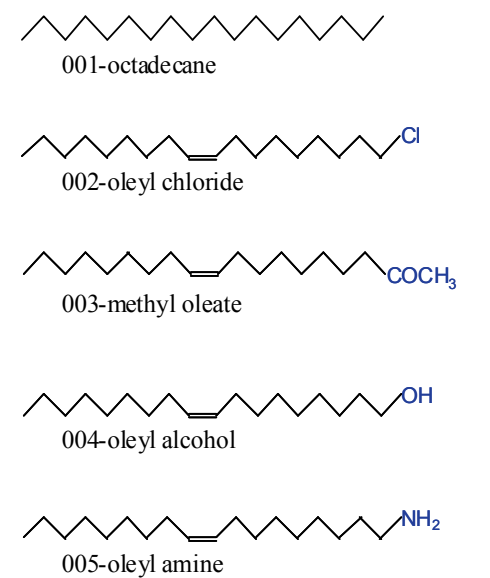

005-oleyl amine

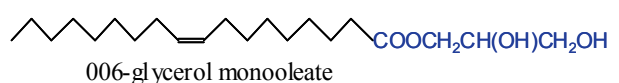

006-glycerol monooleate

007-diglycerol monooleate
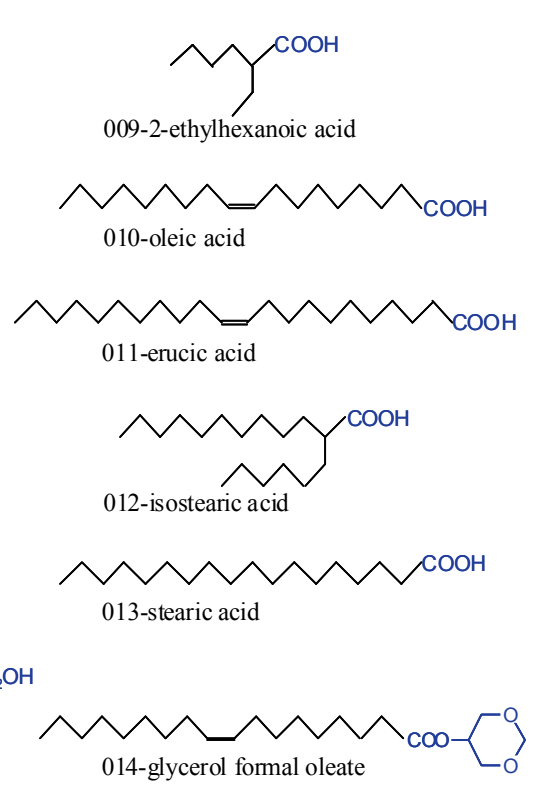

011-erucic acid
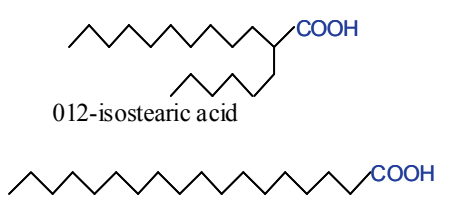

013 -stearic acid

014-glycerol formal oleate

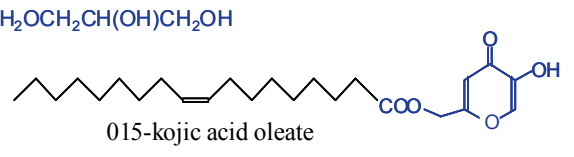

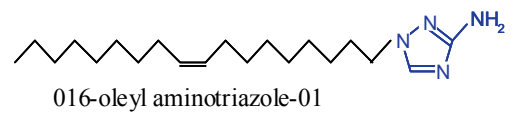

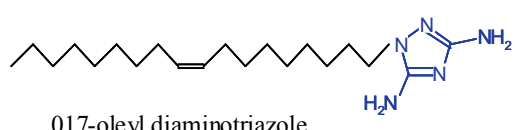

017-oleyl diaminotriazole

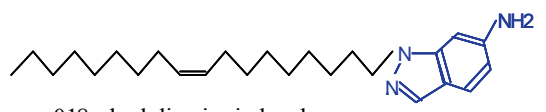

018-oleyl diaminoindazole
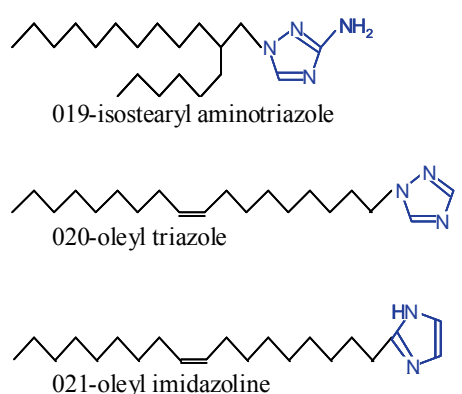

021-oleyl imidazoline

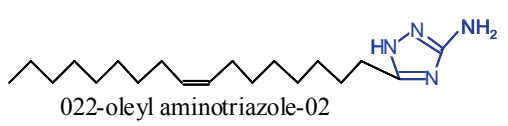

Figure 2 Test compounds

Detailed theory for QSPR analysis has been given elsewhere ${ }^{9)}$. In the present work, the analysis was carried out in the following procedures. Firstly, an optimum molecular structure for each additive is obtained by a software of Winmostar, which is mainly developed based on the semi-empirical molecular orbital theory ${ }^{26,27)}$. Then, based on the structural information, the structural descriptors for each molecule, such as electronic effect, hydrophobicity, threedimensional effect, were extracted and analyzed by a commercial available software MS-QSAR (Accelryth Inc.). Finally, modeling and optimization was perform to generate a correlative expression that related the properties and the structural descriptors was made by Chemish based on the evaluation results of $\mu$ on the iron surface in lubricants with various additives ${ }^{28,29)}$. The multiple regression analysis was carried out to generate normalized numerical values to evaluate the degree of influence of each descriptor.

Details about the SFG principle have been given elsewhere ${ }^{18-23)}$. In the present work, a Ti:sapphire fs laser oscillator (MaiTai) and a regenerative amplifier (SpiteFire PRO) pumped by a Nd:YLF laser (EMPower) can generate a $2.2 \mathrm{~mJ}$ pulse at $800 \mathrm{~nm}$ with a $120 \mathrm{fs}$ duration at a repetition rate of $1 \mathrm{kHz}$. Half of the output was used to pump an optical parametric amplifier (OPA) system (TOPAS) to generate IR pulses tunable from 2.5 to $10 \mu \mathrm{m}$ with a spectral width of ca. $250 \mathrm{~cm}^{-1}$. Remaining output from the amplifier was sent to a home-made spectral shaper to generate a narrow-band pulse (ca. $10 \mathrm{~cm}^{-1}$ ) at $800 \mathrm{~nm}$ to improve the spectral resolution. The broad-band fs IR pulse and the narrow-band visible pulse were overlapped on the sample surface with incident angles of 50 and $70^{\circ}$, respectively. Vibrationally resolved SFG spectra were acquired with a CCD detector attached to a spectrograph.

The lubricant was spin-coated on a polished SUJ-2 plate surface at $2000 \mathrm{rpm}$ and dried in air. SFG spectra were mainly recorded in the $\mathrm{C}-\mathrm{H}$ stretching $(3,000 \sim 2,800$ $\mathrm{cm}^{-1}$ ) region under different polarization combinations of $s$-SFG, $s$-visible, $p$-infrared (denoted as $s s p$ ).

\section{Results and discussion}

3.1. Friction performance of test compounds

Figure 3 shows the reciprocating friction test results of (1) pure PAO and (2) PAO with 1.0 mass\% 016-oleyl aminotriazole-01 under different motion rates. It was found that the $\mu$ values were almost independent on the motion rate of the test ball. In the present work, $\mu$ were measured at $5.0 \mathrm{~Hz}$.

It is interesting to note the $\mu$ decreases from 0.197 (PAO) to 0.108 (PAO +1.0 mass $\%$ 016-oleyl aminotriazole-01), indicating a positive effect of 016oleyl aminotriazole-01 on the friction reduction. The friction performances of all additives on the iron surface have been evaluated by the same method and observed $\mu$ are summarized in Table 2. It is known from the results that these additives show different effects on $\mu$. 
(1) Additive free (PAO)
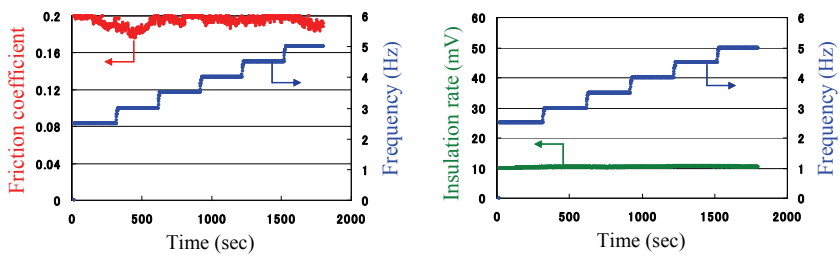

(2) $1.0 \mathrm{wt} \%$ 016-Oleyl aminotriazole-01 in PAO
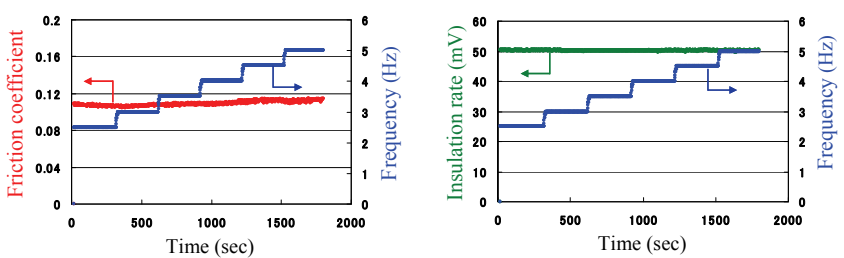

Figure 3 The reciprocating friction test result of (1) PAO; (2) +1.0 mass\% 016-oleyl aminotriazole- 01

Table 2 Friction coefficient of each additive added to $\mathrm{PAO}$

\begin{tabular}{lc}
\hline \multicolumn{1}{c}{ Additives } & Friction coefficient \\
\hline 000-additive free (PAO) & 0.197 \\
001-octadecane & 0.197 \\
002-oleyl chloride & 0.191 \\
003-methyl oleate & 0.169 \\
004-oleyl alcohol & 0.172 \\
005-oleyl amine & 0.152 \\
006-glycerol monooleate & 0.132 \\
007-diglyceryl monooleate & 0.142 \\
008-oleyl amide & 0.129 \\
009-2-ethylhexanoic acid & 0.148 \\
010-oleic acid & 0.108 \\
011-erucic acid & 0.105 \\
012-isostearic acid & 0.135 \\
013-stearic acid & 0.090 \\
014-glycerol formal oleate & 0.133 \\
015-kojic acid oleate & 0.110 \\
016-oleyl aminotriazole-01 & 0.108 \\
017-oleyl diaminotriazole & 0.101 \\
018-oleyl aminoindazole & 0.138 \\
019-isostearyl aminotriazole & 0.132 \\
020-oleyl triazole & 0.134 \\
021-oleyl imidazole & 0.141 \\
022-oleyl aminotriazole-02 & 0.104 \\
\hline
\end{tabular}

013-stearic acid shows most positive effect $(\mu=0.090)$ while the non-polar molecule of 001-octadecan gives the almost identical $\mu(0.197)$ to that of pure PAO. The fiction reduction behavior strongly depends on the chemical natures of the additives.

On the other hand, it is interesting to note the insulation rate increases from $10 \mathrm{mV}$ (PAO) to $50 \mathrm{mV}$ (PAO + 1.0 mass\% 016-oleyl aminotriazole-01), indicating a positive effect of 016-oleyl

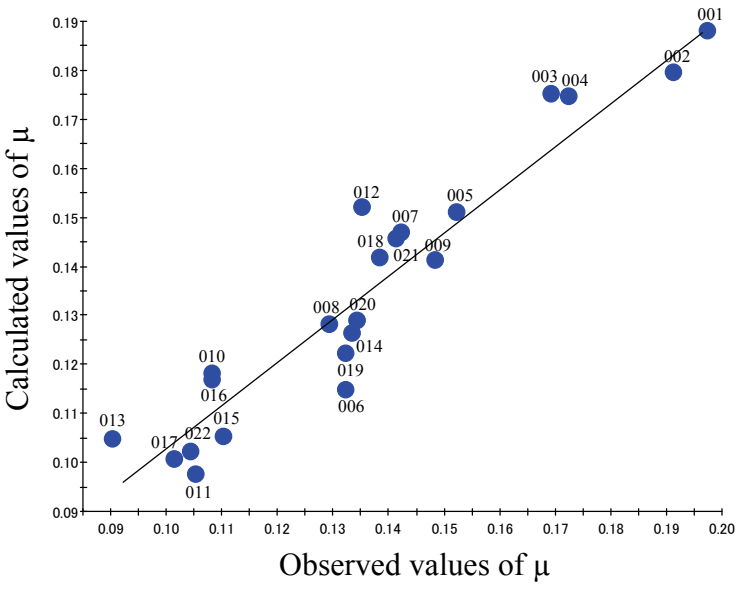

Figure 4 Calculated and observed values of friction coefficient

aminotriazole- 01 on the insulation rate increase. These results suggest that additives with an order adsorption layer on the iron surface have a positive effect on friction reduction.

\subsection{QSPR analysis results}

To understand the relationship between the molecular structures of the additives with their friction reduction activities, multiple regression analysis has been carried out for $\mu$ and structural descriptors of each additive based on the QSPR method shown above. More than 200 descriptors are extracted and their correlations with $\mu$ have been carefully analyzed. A linear relationship (Figure 4, Eq. 1) has been obtained between the observed $\mu$ and the calculated $\mu$ based on the correlation analysis.

$$
\begin{aligned}
& {[\mu]=}-0.002[\text { H-Bond-Donor }]-0.006[\text { E-State-dO }] \\
&-0.007[\text { E-State-ssNH] }-1.286[\text { HOMO energy }] \\
&-0.001[\text { Dipole } \mathrm{m}-\mathrm{X}]-0.004[\text { Shadow length-LX] } \\
&+0.040[\text { Shadow area-YZ }]-0.140 \\
& R^{2}=0.908
\end{aligned}
$$

In the following, we will briefly discuss the contribution of these structural descriptors to the friction reduction based on Eq. (1).

\subsubsection{Influence of hydrogen bond}

First, we will discuss the descriptors related with hydrogen bonding. In the present QSPR analysis (Eq. $1)$, the descriptor term of [H-Bond-Donor] means the number of donor atoms forming hydrogen bond while that of [E-State-dO] and [E-State-ssNH] descriptors correlate with oxygen atom with $\mathrm{C}=\mathrm{O}$ binding and nitrogen atom with two $\mathrm{N}-\mathrm{H}$ bindings in the molecule, respectively. Based on the above descriptors, Figure 5 schematically shows some examples of the hydrogen bonding interactions with the iron surface. It is expected that the additives with a carbonyl, and/or amine group 


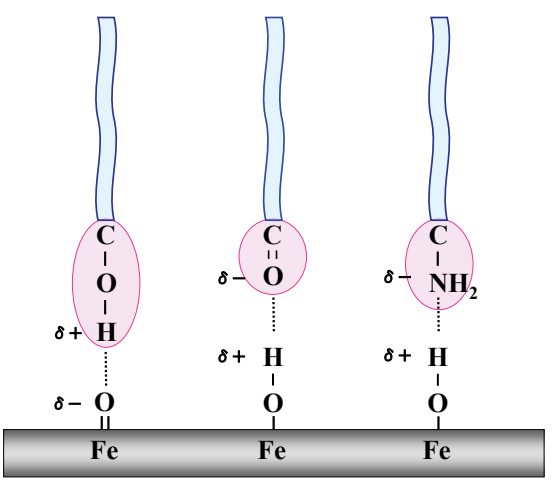

Figure 5 Adsorption image by the hydrogen bond of the compounds to the iron substrate

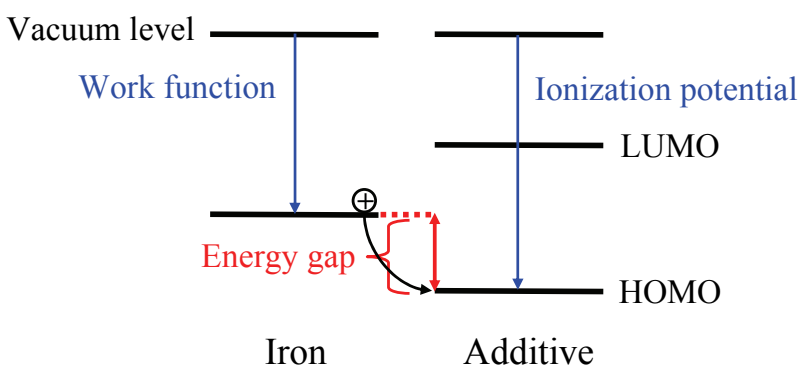

Figure 6 Influence of HOMO energy level

can easy to form the hydrogen bonding with the iron surface. By the hydrogen bonding interaction, these additives can strongly adsorb on the iron surface, and thus consequently make $\mu$ lower.

\subsubsection{Influence of HOMO energy level}

Another important descriptor in Eq. 1 is [HOMO energy] which means the energy of the highest occupied molecular orbital (HOMO). Figure 6 exemplified the influence of HOMO level. It is assumed that the iron and additive molecule work as electron hole donor and acceptor, respectively, in the adsorption process. As the additive molecule's HOMO level become higher, the energy gap between the molecule's HOMO level and the iron's work function becomes smaller and the interaction between the additive and the iron surface will also become stronger. Thus, a higher [HOMO energy] value of additive will lead to a strong interaction with the iron surface and can facilitate the formation of an adlayer on the iron surface which can reduce the friction on the iron surface.

\subsubsection{Influence of dipole moment and length of the} molecule

Additive molecules with large dipole moment usually contain highly polarized hydrophilic group and/or long lipophilic group. In fact, two descriptors in Eq. 1 correlate with the effect. Assuming that the

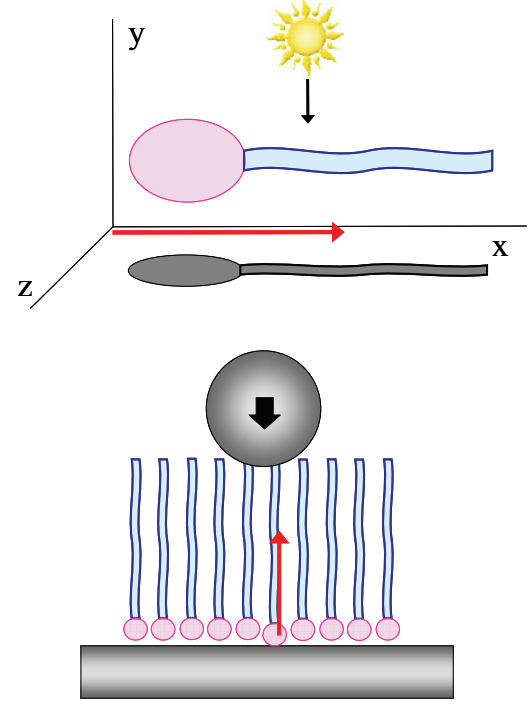

Figure 7 Effect image of dipole moment and molecule length to the iron plate
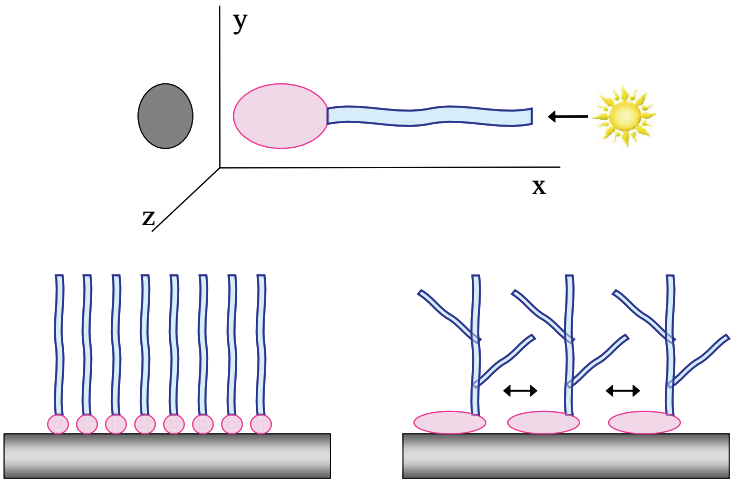

Figure 8 Coating image of the iron plate by friction modifiers

longest axis of the molecule is located on the $x$-axis direction, [Dipole M-X] and [Shadow length-LX] descriptors correspond the dipole moment of molecule in the $x$-direction and length of the molecular shadow on the $x-z$ plane, respectively. It is inferred that superior additives tend to be strongly adsorbed to the iron surface ( $y-z$ plane) through the hydrophilic group and orientate the lipophilic group perpendicular to the surface. As shown in Figure 7, when these additives are aggregated to a layer in a high density, the possibility of other molecules interacting with the iron surface will be largely reduced.

3.2.4. Influence of the projection area of the molecule

As shown in Figure 8, it is predicted that an additive with a highly branched structure is hard to form a densely packed adlayer on the iron surface and consequently, is difficult to get lower $\mu$. This influence can be reflected by [Shadow area-YZ] descriptor in Eq. 
(1). For example, stearic acid gives the smallest $\mu(0.090)$ in the additives while isostearic acid with branched structure shows a similar $\mu(0.135)$ with that of pure PAO.

\subsection{SFG observation of the molecule adsorbed on the} iron surface

Based on the discussion above, additives with lower $\mu$ are expected to organize as an adlayer in a high density and orientate their lipophilic groups perpendicularly on the iron surface. In the present section, the adsorption structures of the additives on the iron surface were directly evaluated by SFG measurements.

Figure 9 shows the $s s p$-polarized SFG spectra in the $\mathrm{C}-\mathrm{H}$ region on the polished iron surface in $\mathrm{PAO}$ containing with 1.0 mass $\%$ additives. Three dominant peaks were observed at about 2940, 2880 and $2850 \mathrm{~cm}^{-1}$, which are assigned to Fermi resonance of the symmetric $\mathrm{C}-\mathrm{H}$ stretching of methyl $\left(\mathrm{CH}_{3}\right)$ group, the symmetric $\mathrm{C}-\mathrm{H}$ stretching mode of the $\mathrm{CH}_{3}$ group and the symmetric $\mathrm{C}-\mathrm{H}$ stretching mode of the methylene $\left(\mathrm{CH}_{2}\right)$ group, respectively ${ }^{18-22)}$. It is known that the $\mathrm{SFG}$ signals from $\mathrm{CH}_{2}$ groups are almost forbidden due to the local symmetry restriction if the alkyl chains are orientated in the all-trans conformation. On the other hand, if the gauche conformation is present in the alkyl chain, the local symmetry in the alkyl chain is broken and thus SFG signals from $\mathrm{CH}_{2}$ are also available. Appearance of the peak at $2850 \mathrm{~cm}^{-1}$ in the SFG spectra (Figure 9) indicates that the alkyl chains of these additive adlayers contain certain gauche defects. To quantitatively understand the influence of the structure difference in the adlayers, the SFG spectra were analyzed by the following equation:

$$
I_{S F G} \propto\left|\sum_{v} \frac{A_{v}}{\omega_{I R}-\omega_{v}+i \Gamma_{v}} e^{i \phi_{v}}+\chi_{N R}^{(2)}\right|^{2}
$$

where $A_{v}$ and $\Gamma_{v}$ are the amplitude and the damping constant, respectively, of the vibrational mode $v$ at a frequency $\omega_{v}$ with a phase angle $\left(\varphi_{v}\right)$ with respect to non-resonant signal $\left(\chi_{N R}^{(2)}\right)$. The amplitude ratio between $\mathrm{CH}_{2}$ and $\mathrm{CH}_{3}$ modes $\left(R=A_{\mathrm{CH} 2} / A_{\mathrm{CH} 3}\right)$ from the SFG observation has been employed to discuss the influence of the conformation order of hydrocarbon chains $^{19-22)}$. The lower the $R$ value is observed, the less gauche defects is present in the adlayer. As shown in Figure 10, the PAO shows a large $\mathrm{CH}_{2}$ peak in comparison with $\mathrm{CH}_{3}(R=0.76)$. As 1.0 mass\% 016-oleyl aminotriazole-01 was added into PAO, $R$ decreased to 0.34 , indicating a higher conformation order of the additive on the iron surface to that in PAO. On the same time, $\mu$ decreased 0.197 to 0.108 . On the other hand, addition of 002-oleyl chloride shows an increase of $R(0.95)$ and a similar $\mu(0.191)$ in comparison with those observed in pure PAO.

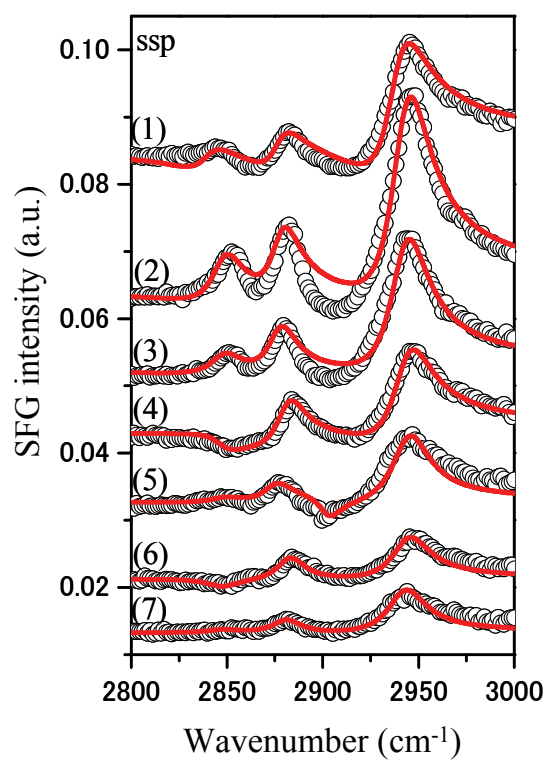

Figure 9 The $s s p$-polarized SFG spectra of various additives added to PAO on the iron surface

(1) PAO;

(2) +1.0 mass $\%$ 002-oleyl chloride;

(3) +1.0 mass $\%$ 005-oleyl amine;

(4) +1.0 mass $\%$ 012-isostearic acid;

(5) +1.0 mass $\%$ 016-oleyl aminotriazole- 01 ;

(6) +1.0 mass $\%$ 006-glycerol monooleate;

(7) +1.0 mass $\%$ 010-oleic acid

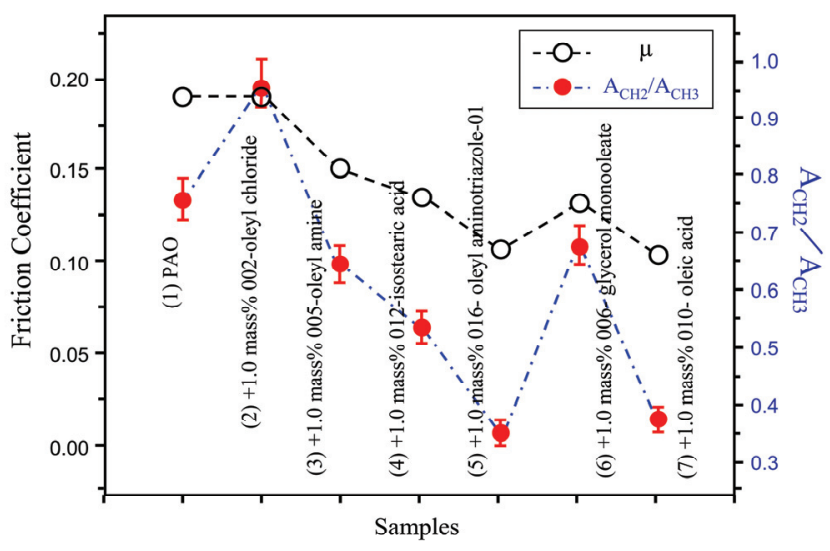

Figure 10 Friction coefficient and SFG intensity ratio observed for different additives added to PAO

Figure 10 summarized $R$ values observed from SFG measurement and $\mu$ obtained from friction experiments for several additives. It is interesting to note that there exist certain correlations between the $R$ and $\mu$. It is found that additive adlayers with higher conformation orders on the iron surface usually show lower $\mu$, i.e., the 
higher friction reduction effect.

These results qualitatively agree well with the results obtained by the QSPR measurement and suggest that additives with an order adsorption layer on the friction surface have a positive effect on friction reduction. Detailed studies are still in progress. The present work also indicates that SFG is useful for understanding the relation between the adsorption structure/conformation and friction behaviors of the additives on the tribological interface.

\section{Conclusions}

In the present study, mechanism governing the friction performance of ashless-type friction modifiers has been studied by using friction evaluation, QSPR analysis and SFG measurement. A correlative relation between the friction performances with the molecular structure was obtained. This general relation was used to understand the structural feature of high-performance friction modifiers. It was inferred that molecules that formed an ordered adlayer on the friction surface showed a superior friction reducing effect.

\section{References}

[1] Akiyama, K., Kawai, H. and Sugiyama, S., "Development of Fuel Economy 5W-20 Gasoline Engine Oil," Toyota Technical Review, 47, 2, 1997 , 20-25. (in Japanese)

[2] Sagawa, T., Ishikawa, T., Nakamura, K., Ueno, T., Ando, T. and Ishikawa, M., "Development of 0w-20 Ilsac Gf-3 Gasoline Engine Oil," Society of Automotive Engineers, 2002-01-1636, 2002.

[3] Muraki, M. and Wada, H., "Frictional Properties of Organo Molybdenum Compounds in Presence of ZnDTP under Sliding Condition," Journal of Japanese Society of Tribologists, 38, 10, 1993, 919-926. (in Japanese)

[4] Yamamoto, Y. and Gondo, S., "Friction and Wear Characteristics of Molybdenum Dithiocarbamate and Molybdenum Dithiophosphate," Tribology Transaction, 32, 2, 1989, 251-257.

[5] Yaguchi, A. and Inoue, K., "Development and Field Test Performance of Fuel Efficient SAE 5w-20 Oils," Society of Automotive Engineers, 952341, 1995.

[6] Akiyama, T., Ueda, F., Kurono, K., Kawai, H. and Sugiyama, S., "Development of Fuel Economy 5W-20 Gasoline Engine Oil," Proc. Society of Automotive Engineers of Japan, Annual Congress Autumn, No.975, 9740109, 1997. (in Japanese)

[7] Lundgren, S. M., Ruths, M., Danerlov, K. and Persson, K., "Effect of Unsaturation on Film Structure and Friction of Fatty Acid in a Model Base Oil," Journal of Colloid and Interface Science, 326, 2008, 530-536.

[8] Lundgren, S. M., Persson, K., Mueller, G., Kronberg, B., Clarke, J., Chtaib, M. and Claesson,
P. M., "Unsaturated Fatty Acids in Alkane Solution: Adsorption to Steel Surfaces," Langmuir, 23, 2007, 10598-10602.

[9] Gasteiger, J. and Engel, T., "Chemoinformatics A Textbook," Wiley, New Jersey, 2003.

[10] Tsubouchi, T., Hata, H. and Yoshida, Y., "Optimisation of Molecular Structure for Traction Fluids," Lubrication Science, 16, 4, 2004, 393-403.

[11] Tsubouchi, T. and Hata, H., "Traction Fluids," Journal of Japanese Society of Tribologists, 42, 3, 1997, 193-198. (in Japanese)

[12] Koshima, H. and Tsubouchi, T., "Study on the Influencing Factors of Phenyl Salicylates to the High-Temperature Detergency," Proc. Abstracts of International Tribology Conference Kobe, Satellite Forum Tribochemistry Nara, 2005, 33.

[13] Koshima, H., Tsubouchi, T. and Hisaeda, Y., "The Study of Influencing Factors of Phenyl Salicylates to the High-Temperature Detergency," Triborogy Online, 3, 3, 2008, 195-199.

[14] Koshima, H. and Ichihashi, T., "Antishudder Performance of Low-Molecular-Weight Alkenylsuccinimides," Proc. Society of Automotive Engineers of Japan, Annual Congress Autumn, 130, 7, 20075638, 2007. (in Japanese)

[15] Koshima, H., Tsubouchi, T., Ichihashi, T. and Hisaeda, Y., "Antishudder Performance of Low-Molecular-Weight Alkenylsuccinimides," Tribology Online, 3, 6, 2008, 328-332.

[16] Shen, Y. R., "The Principles of Nonlinear Optics," John Wiley \& Sons, Inc., New York, 1984.

[17] Bain, C. D., "Sum-Frequency Vibrational Spectroscopy of the Solid/Liquid Interface," J. Chem. Soc. Faraday Trans., 91, 1995, 1281-1296.

[18] Ye, S. and Osawa, M., "Molecular Structures on Solid Substrates Probed by Sum Frequency Generation (SFG) Vibration Spectroscopy," Chem. Lett., 38, 5, 2009, 386-391.

[19] Guyot-Sionnest, P., Hunt, J. H. and Shen, Y. R., "Sum-Frequency Vibrational Spectroscopy of a Langmuir Film: Study of Molecular Orientation of a Two-Dimensional System,” Phys. Rev. Lett., 59, 1987, 1597-1600.

[20] Miranda, P. D., Pflumio, V., Saijo, H. and Shen, Y. R., "Chain-Chain Interaction between Surfactant Monolayers and Alkanes or Alcohols at Solid/Liquid Interfaces," J. Am. Chem. Soc., 120, 1998, 12092-12099.

[21] Conboy, J. C., Messmer, M. C. and Richmond, G. L., "Dependence of Alkyl Chain Conformation of Simple Ionic Surfactants on Head Group Functionality as Studied by Vibrational Sum-Frequency Spectroscopy,” J. Phys. Chem. B., 101, 1997, 6724-6733.

[22] Ward, R. N., Duffy, D. C., Davies, P. B. and Bain, C. D., "Sum-Frequency Spectroscopy of Surfactants Adsorbed at a Flat Hydrophobic Surface,” J. Phys. Chem., 98, 1994, 8536-8542. 
[23] Holman, J., Davis, P. B., Nishida, T., Ye, S. and Neivandt, D. J., "Sum Frequency Generation from Langmuir-Blodgett Multilayer Films on Metal and Dielectric Substrates," J. Phys. Chem. B, 109, 2005, 18723-18732.

[24] Nishida, T., Johnson, C. M., Holman, J., Osawa, M., Davies, P. B. and Ye, S., "Optical Sum-Frequency Emission from Langmuir-Blodgett Films of Variable Thickness: Effects of the Substrate and Polar Orientation of Fatty Acids in the Films," Phys. Rev. Lett., 96, 2006, 077402.

[25] Duffy, D. C., Friedmann, A., Boggis, S. A. and Klenerman, D., "Surface Vibrational Spectroscopy of Lubricants Adsorbedat the Iron-Water Interface," Langmuir, 14, 1998, 6518-6527.

[26] http://winmostar.com

[27] Senda, N., "Development of Molecular Calculation Support System Winmostar (9)," Society of Computer Chemistry Japan, Proceedings of Annual Congress Autumn, 1P02, 2008. (in Japanese)

[28] http://www.cheminfonavi.co.jp/chemish/

[29] Tanada, T., Arakawa, M., Nishimura, R. and Funatsu, K., "Development of Material Design Program Based on Chemometrics," Journal of Computer Aided Chemistry, 1, 2000, 35-46.

\section{Nomenclature}

$[\mu] \quad$ Friction coefficient

[H-Bond-Donor] The number of donor atoms forming hydrogen bond

[E-State-dO] The numerical value for oxygen, with double bond, of both the topological environment and the electronic interactions due to other atoms in the molecule

[E-State-ssNH] The numerical value for nitrogen bonded to hydrogen, with two single bond, of both the topological environment and the electronic interactions due to other atoms in the molecule

[HOMO energy] The energy of the highest occupied molecular orbital

[Dipole M-X] The dipole moment of molecule in the $x$-direction

[Shadow length-LX] The length of molecular shadow on the $x-z$ plane

[Shadow area-YZ] The area of molecular shadow on the $y-z$ plane 